$(-65 \mathrm{mV})$ are the reversal potentials for excitatory and inhibitory currents. Changing $E_{\mathrm{e}}$ and $E_{\mathrm{i}}$ by $\pm 10 \mathrm{mV}$ did not change the time course of any conductance significantly.

Skewness, a measure of the asymmetry based on the third central moment of a distribution, is defined as $m_{3} / m_{2}^{3 / 2}$, where $m_{1}=\Sigma\left(r_{f} f\right), m_{2}=\Sigma\left(r_{f}\left(f-m_{1}\right)^{2}\right), m_{3}=$ $\Sigma\left(r_{f}\left(f-m_{1}\right)^{3}\right)$ and $r_{f}=R_{f} / \Sigma\left(R_{f}\right) . R_{f}$ is the synaptic current at $-70 \mathrm{mV}$, averaged between 15 and $35 \mathrm{~ms}$ after the onset of a tone of frequency $f$, with $f$ in units of octaves above $0.5 \mathrm{kHz}$. The sums are over all frequencies in the synaptic TRF at $60 \mathrm{~dB}$. Skewness was calculated only for neurons whose synaptic TRFs lay entirely within the probed frequency range.

Received 10 February; accepted 7 May 2003; doi:10.1038/nature01796.

1. Mendelson, J. R. \& Cynader, M. S. Sensitivity of cat primary auditory cortex (AI) neurons to the direction and rate of frequency modulation. Brain Res. 327, 331-335 (1985).

2. Schreiner, C. E., Read, H. L. \& Sutter, M. L. Modular organization of frequency integration in primary auditory cortex. Annu. Rev. Neurosci. 23, 501-529 (2000).

3. Ricketts, C., Mendelson, J. R., Anand, B. \& English, R. Responses to time-varying stimuli in rat auditory cortex. Hearing Res. 123, 27-30 (1998).

4. Merzenich, M. M., Knight, P. L. \& Roth, G. L. Representation of cochlea within primary auditory cortex in the cat. J. Neurophysiol. 38, 231-249 (1975).

5. Sally, S. L. \& Kelly, J. B. Organization of auditory cortex in the albino rat: sound frequency. J. Neurophysiol. 59, 1627-1638 (1988).

6. Suga, N. Functional properties of auditory neurones in the cortex of echo-locating bats. J. Physiol. 181, 671-700 (1965).

Shamma, S. A., Fleshman, J. W., Wiser, P. R. \& Versnel, H. Organization of response areas in ferret primary auditory cortex. J. Neurophysiol. 69, 367-383 (1993).

8. Nelken, I. \& Versnel, H. Responses to linear and logarithmic frequency-modulated sweeps in ferret primary auditory cortex. Eur. J. Neurosci. 12, 549-562 (2000).

9. Calford, M. B. \& Semple, M. N. Monaural inhibition in cat auditory cortex. J. Neurophysiol. 73, 1876-1891 (1995)

10. Brosch, M. \& Schreiner, C. E. Time course of forward masking tuning curves in cat primary auditory cortex. J. Neurophysiol. 77, 923-943 (1997)

11. Clopton, B. M. \& Winfield, J. A. Unit responses in the inferior colliculus of rat to temporal auditory patterns of tone sweeps and noise bursts. Exp. Neurol. 42, 532-540 (1974).

12. Britt, R. \& Starr, A. Synaptic events and discharge patterns of cochlear nucleus cells. II. Frequencymodulated tones. J. Neurophysiol. 39, 179-194 (1976).

13. Poon, P. W. \& Yu, P. P. Spectro-temporal receptive fields of midbrain auditory neurons in the rat obtained with frequency modulated stimulation. Neurosci. Lett. 289, 9-12 (2000).

14. Kowalski, N., Depireux, D. A. \& Shamma, S. A. Analysis of dynamic spectra in ferret primary auditory cortex. II. Prediction of unit responses to arbitrary dynamic spectra. J. Neurophysiol. 76, 3524-3534 (1996).

15. deCharms, R. C., Blake, D. T. \& Merzenich, M. M. Optimizing sound features for cortical neurons. Science 280, 1439-1443 (1998).

16. Schnupp, J. W., Mrsic-Flogel, T. D. \& King, A. J. Linear processing of spatial cues in primary auditory cortex. Nature 414, 200-204 (2001).

17. De Ribaupierre, F., Goldstein, M. H. Jr \& Yeni-Komshian, G. Intracellular study of the cat's primary auditory cortex. Brain Res. 48, 185-204 (1972).

18. Volkov, I. O. \& Galazjuk, A. V. Formation of spike response to sound tones in cat auditory cortex neurons: interaction of excitatory and inhibitory effects. Neuroscience 43, 307-321 (1991).

19. Ojima, H. \& Murakami, K. Intracellular characterization of suppressive responses in supragranular pyramidal neurons of cat primary auditory cortex in vivo. Cereb. Cortex 12, 1079-1091 (2002).

20. Mehta, M. R., Quirk, M. C. \& Wilson, M. A. Experience-dependent asymmetric shape of hippocampal receptive fields. Neuron 25, 707-715 (2000).

21. Engert, F., Tao, H. W., Zhang, L. I. \& Poo, M. Moving visual stimuli rapidly induce direction sensitivity of developing tectal neurons. Nature 419, 470-475 (2002).

22. Rao, R. P. N. \& Sejnowski, T. J. in Advances in Neural Information Processing Systems 12 (eds Solla, S. A., Leen, T. K. \& Muller, K. R.) 164-170 (MIT Press, Cambridge, Massachusetts, 2000).

23. Zhang, L. I., Bao, S. \& Merzenich, M. M. Persistent and specific influences of early acoustic environments on primary auditory cortex. Nature Neurosci. 4, 1123-1130 (2001).

24. Zhang, L. I., Tao, H. W., Holt, C. E., Harris, W. A. \& Poo, M. A critical window for cooperation and competition among developing retinotectal synapses. Nature 395, 37-44 (1998).

25. Moore, C. I. \& Nelson, S. B. Spatio-temporal subthreshold receptive fields in the vibrissa representation of rat primary somatosensory cortex. J. Neurophysiol. 80, 2882-2892 (1998)

26. Zhu, J. J. \& Connors, B. W. Intrinsic firing patterns and whisker-evoked synaptic responses of neurons in the rat barrel cortex. J. Neurophysiol. 81, 1171-1183 (1999).

27. Margrie, T. W., Brecht, M. \& Sakmann, B. In vivo, low-resistance, whole-cell recordings from neurons in the anaesthetized and awake mammalian brain. Pflugers Arch. 444, 491-498 (2002).

28. Borg-Graham, L. J., Monier, C. \& Fregnac, Y. Visual input evokes transient and strong shunting inhibition in visual cortical neurons. Nature 393, 369-373 (1998).

29. Hirsch, J. A., Alonso, J. M., Reid, R. C. \& Martinez, L. M. Synaptic integration in striate cortical simple cells. J. Neurosci. 18, 9517-9528 (1998)

30. Anderson, J. S., Carandini, M. \& Ferster, D. Orientation tuning of input conductance, excitation, and inhibition in cat primary visual cortex. J. Neurophysiol. 84, 909-926 (2000).

Supplementary Information accompanies the paper on www.nature.com/nature.

Acknowledgements This work was supported by the Howard Hughes Medical Institute, the John C. and Edward Coleman Fund, the MacDonnell Foundation, the National Institutes of Health and the Sandler Fund.

Competing interests statement The authors declare that they have no competing financial interests.

Correspondence and requests for materials should be addressed to L.I.Z. (lizhang@phy.ucsf.edu).

\section{Glutamate-receptor-mediated encoding and retrieval of paired-associate learning}

\section{Day, R. Langston \& R. G. M. Morris}

Laboratory for Cognitive Neuroscience, Division and Centre for Neuroscience, The University of Edinburgh, 1 George Square, Edinburgh EH8 9JZ, UK

Paired-associate learning is often used to examine episodic memory in humans ${ }^{1}$. Animal models include the recall of foodcache locations by scrub jays ${ }^{2}$ and sequential memory ${ }^{3,4}$. Here we report a model in which rats encode, during successive sample trials, two paired associates (flavours of food and their spatial locations) and display better-than-chance recall of one item when cued by the other. In a first study, pairings of a particular foodstuff and its location were never repeated, so ensuring unique 'what-where' attributes. Blocking $N$-methyl-D-aspartate receptors in the hippocampus-crucial for the induction of certain forms of activity-dependent synaptic plasticity ${ }^{5,6}$ impaired memory encoding but had no effect on recall. Inactivating hippocampal neural activity by blocking $\alpha$-amino-3hydroxy-5-methyl-4-isoxazole propionic acid (AMPA) receptors impaired both encoding and recall. In a second study, two paired associates were trained repeatedly over 8 weeks in new pairs, but blocking of hippocampal AMPA receptors did not affect their recall. Thus we conclude that unique what-where paired associates depend on encoding and retrieval within a hippocampal memory space ${ }^{7,8}$, with consolidation of the memory traces representing repeated paired associates in circuits elsewhere.

Paired-associate learning typically consists of a study phase of pairs of items (such as word pairs) followed by a test of cued recall. Our new protocol involves training rats in a large arena containing a $7 \times 7$ grid of 49 sand-wells that could be uncovered selectively (Fig. 1). A 'sample' trial (Fig. 1a) consists of the rat leaving one of the start boxes to search for a single uncovered sand-well where it can dig to get a 1-g pellet of flavoured rat chow. A second sample trial is given 2 min later with a different flavour of chow at a different location (Fig. 1b). The following cued-recall trial is given in several ways, but on training trials as a rewarded choice. The recall cue is a $500-\mathrm{mg}$ pellet of one of the two earlier flavours, available in a different start box for a 30-second recollection interval during which it is eaten. The animal is then allowed into the arena, which now has two sand-wells uncovered (L1 and L2; Fig. 1c). If cued with food $\mathrm{F} 1$, the rat is rewarded with a 1-g pellet of $\mathrm{F} 1$ by going to and digging at L1; if cued with F2, it is rewarded with F2 at L2 (see Supplementary Information on counterbalancing). On the next day, a new pair of flavours and spatial locations is chosen as paired associates with an inevitable reuse of both flavours and locations in novel combinations (of which there are 28).

After arena habituation, the rats $(n=8)$ were digging readily for the buried food. The main training then began and, on the measure of first chosen sand-well during a choice trial, better-then-chance performance was observed very early. The rats generally kept their heads near the floor of the arena during sample trials, displaying small lateral head movements reflecting their search for the single, unobtrusive sand-well that was open (see Sample 2 of the QuickTime movie at http://neuroweb-2.dns.ed.ac.uk/video/). On choice trials, the rats ran relatively directly towards one of the two sample sand-wells, indicating recall of location, without displaying these head movements. After only 6 days of training, the average first-

* Present address: GlaxoSmithKline, Centre for Excellence in Drug Discovery, Harlow, Essex, CM19 5AW, UK. 

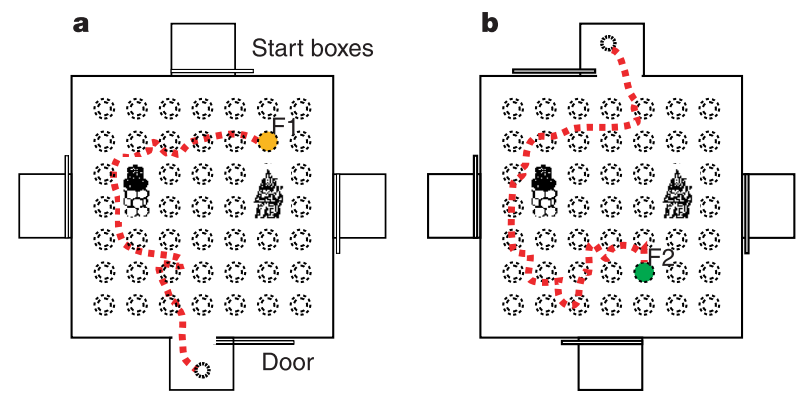

Figure 1 The event arena. a, A rat runs into the arena (dotted line), where it displays lateral head movements to find food 1 ( $F 1$, orange) at the single open sand-well. b. Sample 2 is a different food (F2, green) at a different location. c. The cued-recall choice trial begins with presentation of either of the two sample-trial foods (for example, F1 as

choice performance over the last 5 days was $70.0 \pm 7.6 \%$ (s.e.m.) correct (Fig. 1d), comparable to what is seen in spontaneous alternation.

To check that apparent recall was not mediated by odours emanating from the flavoured foodstuffs, two non-rewarded probe tests were scheduled in which no food was hidden in either of the two sampled sand-wells on choice trials. The animals, together with others first trained with double-sample trials, were trained with the single-presentation sample procedure for a further 10 days of training, and reduced to the most consistent 12 of the original 16 rats ( $n=6$ from each subgroup). The probe tests also offered the opportunity to examine what would happen if a further three sand-wells (locations 3-5) were uncovered that had not been open on the two sample trials of that day. The animals were allowed to dig for up to 60 seconds and we observed above-average digging time at the cued location (Fig. 2a). In a second probe test, the rats were 'miscued' by giving them a novel flavour (F3) as a recall cue at the start of the choice trial. The rats now showed equal preference for the two previously sampled locations (L1, L2) that was significantly higher than for the unsampled locations (Fig. 2b). We conclude that rats can encode a flavour-location association in a single trial and later display cued recall of the paired associate.

This new one-trial paradigm was explicitly designed to investigate whether activation of glutamate receptors ${ }^{9}$ in the hippocampus would differentially mediate encoding and retrieval memory processes. To do this, we examined the selective antagonists 6-cyano-7nitroquinoxaline (CNQX, an antagonist of AMPA receptors) and $\mathrm{D}(-)$-2-amino-5-phosphonopentanoic acid (D-AP5, an antagonist of NMDA ( $N$-methyl-D-aspartate) receptors) by infusing them (or artificial cerebrospinal fluid; aCSF) into the dorsal hippocampus $15 \mathrm{~min}$ before sample trials or $15 \mathrm{~min}$ before choice trials (sample and choice were scheduled $20 \mathrm{~min}$ apart). A within-subjects protocol was used in which each of the 12 rats was trained over 17 days with 11 regular training days (some of which included infusions with aCSF) interspersed with the six drug-infusion conditions (given as non-rewarded probe trials; see the representative sequence in Fig. 3c).

Rats given aCSF infusions showed better-then-chance choice performance irrespective of when the infusions occurred (Fig. 3c, d). Infusion of D-AP5 impaired choice accuracy when infused before sample trials, but not when infused after sample trials but before choice trials. Infusion of CNQX impaired choice accuracy whether given before or after a sample trial. This dissociation was by no means a foregone conclusion, but makes sense considering the role of NMDA receptors in the induction of activity-dependent synaptic plasticity in the hippocampus ${ }^{10}$ (Fig. 3b) and of AMPAmediated fast synaptic transmission in expressing changes in
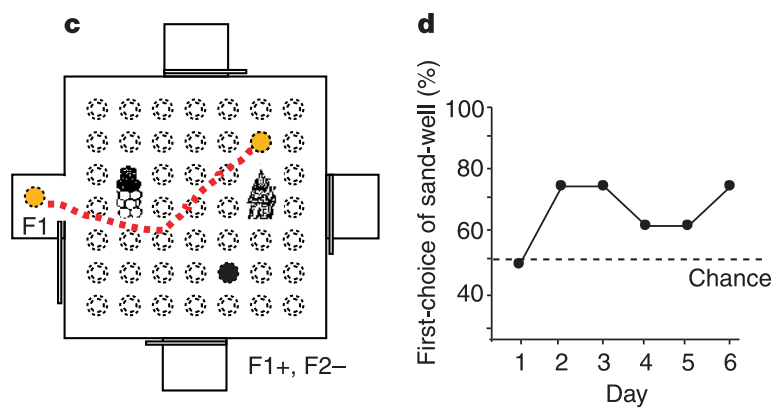

shown) with the rat selectively rewarded for digging at the sand-well containing this same food. d, Rapid learning over 6 days of training. The rats $(n=8)$ performed better than chance (dotted line) on days $2-6(t=2.65$, d.f. $7, P<0.03)$.

synaptic weight ${ }^{11}$ (Fig. 3a, ref. 11).

There are two caveats, both related to the involvement of the hippocampus in spatial memory ${ }^{12,13}$. One is that NMDA receptors might be necessary for encoding the place of a novel sand-well within a familiar environment, as in a water maze ${ }^{14}$, and this encoding might be a prerequisite for forming a flavour-place association. The other is that the deficit in paired-associate recall during hippocampal inactivation by CNQX might be secondary to a disruption of spatial memory. The latter caveat is unlikely because the animals had received extensive exposure to the arena by the time
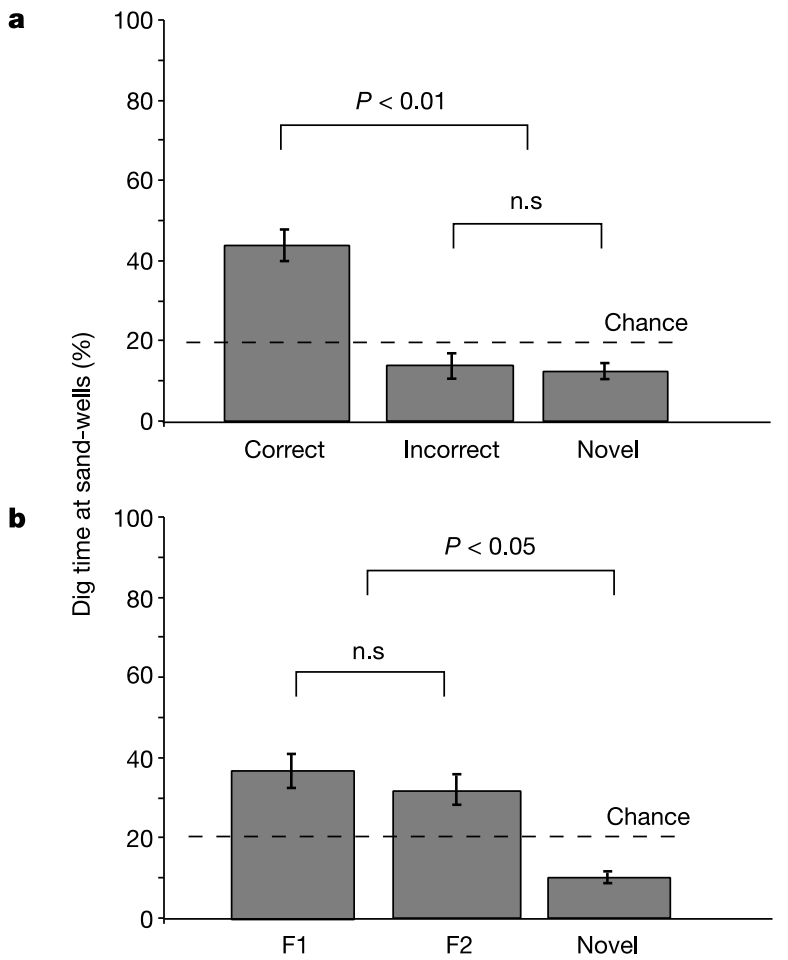

Figure 2 Non-rewarded probe tests. a, Percentage of time spent digging on choice trials was significantly different at the correct (cued), incorrect and novel sand-wells $(F=6.92$, d.f. $2 / 22, P<0.005)$. Orthogonal comparisons revealed that the time spent at the incorrect and novel locations did not differ $(F<1)$, but time spent at the correct location was significantly higher ( $F=13.8$, d.f. $1 / 22, P<0.01)$. b. Choice trials 'miscued' by a flavour not used during the sample trials revealed that time spent at the two sampled locations did not differ $(F<1)$ but was significantly higher than at the novel locations $(F=5.27$, d.f. $1 / 22, P<0.05)$. 
of the drug infusions (2 months), sufficient to allow spatial information to be consolidated outside the hippocampus ${ }^{15}$. We could exclude this second concern if memory retrieval can then bypass this structure. Two flavour-place pairings (apple at location $(1,5)$; brandy at location $(7,5))$ were therefore trained repeatedly over 8 weeks, amid further training on single-trial novel associates. Both paired associates had at least 20 training presentations before the start of non-rewarded probe tests. The training protocol included two sample trials and one choice trial each day, as before, but choice trials in which a total of four sand-wells were generally available (see Supplementary Information). Non-rewarded probe trials included the cued sand-well; a non-cued but previously sampled sand-well; a never rewarded sand-well (always at $(3,3)$ ); and a novel well (Fig. 4a).
The two repeat pairings were gradually learned over the 2-month training period (for example, an increasing proportion of time spent digging at the relevant wells on rewarded choice trials: $F=3.67$, d.f. $2 / 24, P<0.05$; data not shown). The key tests of whether cued recall of 'consolidated' associates would be possible during hippocampal inactivation were conducted in a counterbalanced manner over the next 2 weeks (weeks 9 and 10), interspersed with further training days to maintain performance, with a further probe trial in week 11. Intrahippocampal CNQX had no effect on the ability of rats to navigate appropriately and dig persistently at the correct location when cued with either repeatpair flavour (Fig. 4b). The final probe trials (data not shown) confirmed that the animals could be cued by the relevant flavour to choose, under CNQX or CSF, between just the two repeat-trial
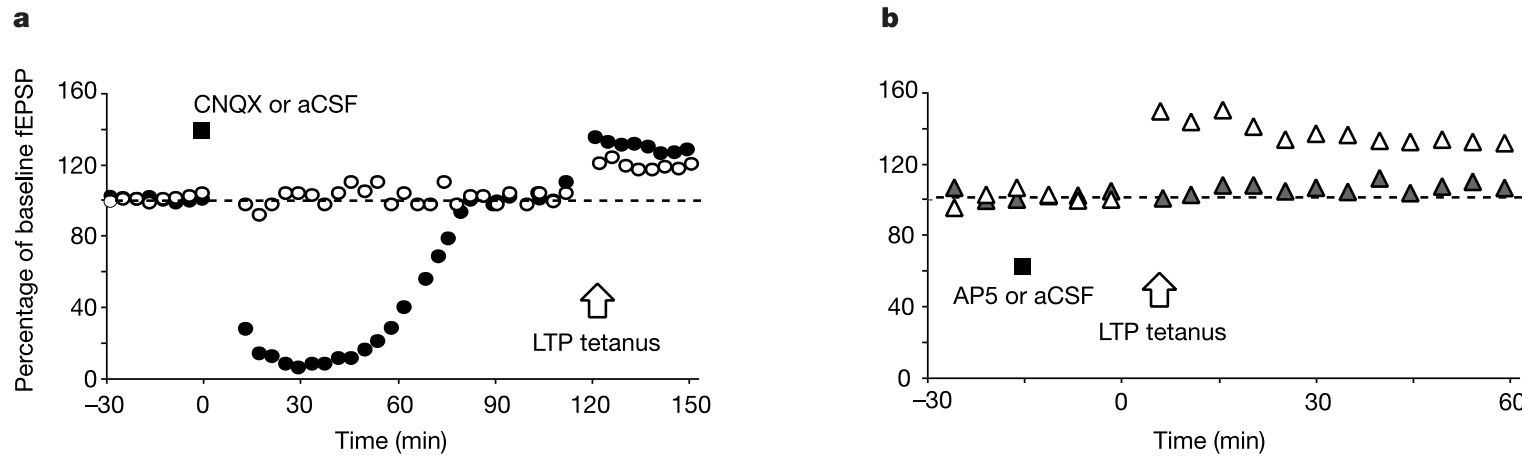

c

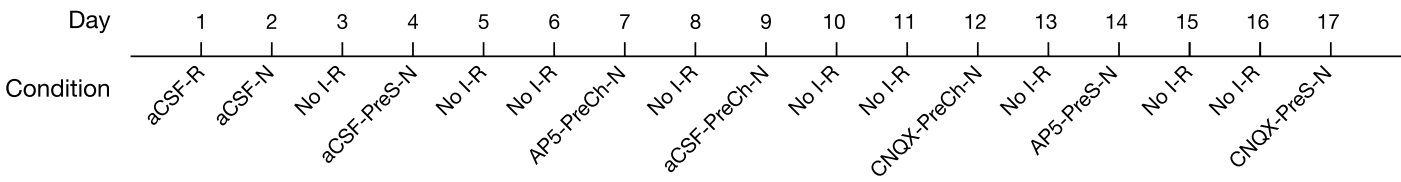

d

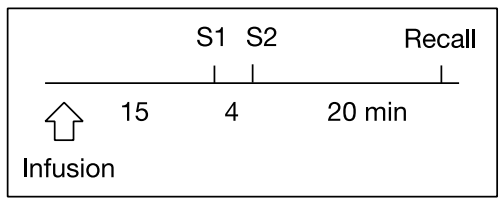

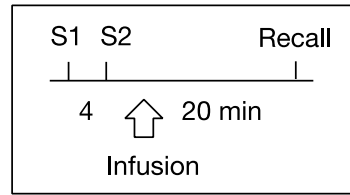

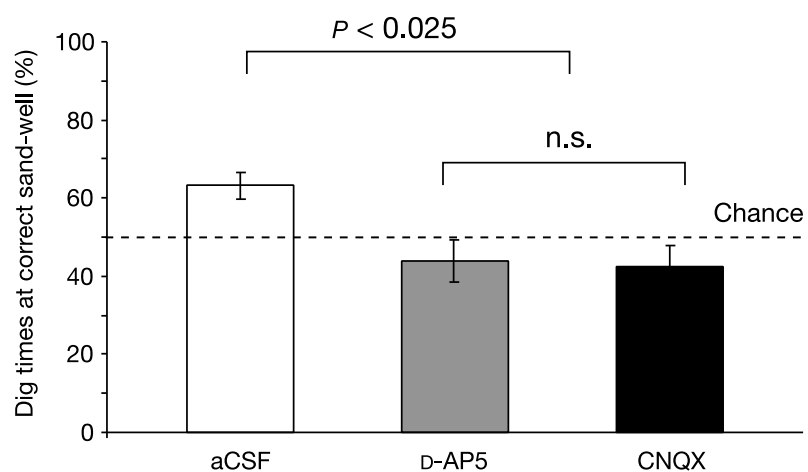

Figure 3 Differential glutamate-receptor dependence of encoding and retrieval.

a, Maximal neural inactivation in the hippocampus occurred within 10-15 min of CNQX infusion and lasted for $\sim 60 \mathrm{~min}$. Open circles, aCSF; filled circles, CNQX. LTP, long-term potentiation. b, D-AP5 infusions did not affect fast synaptic transmission but blocked LTP induction 15 min after infusion. Open triangles, aCSF; filled triangles, D-AP5.

c, Representative sequence of drug treatments for an individual rat across 17 days (counterbalanced with respect to treatment order). D-AP5, CNQX and aCSF indicate bilateral infusion; $R$ and $N$ indicate that a choice trial was rewarded or non-rewarded; PreS and PreCh indicate infusions before sample or choice. Nol signifies no infusion.

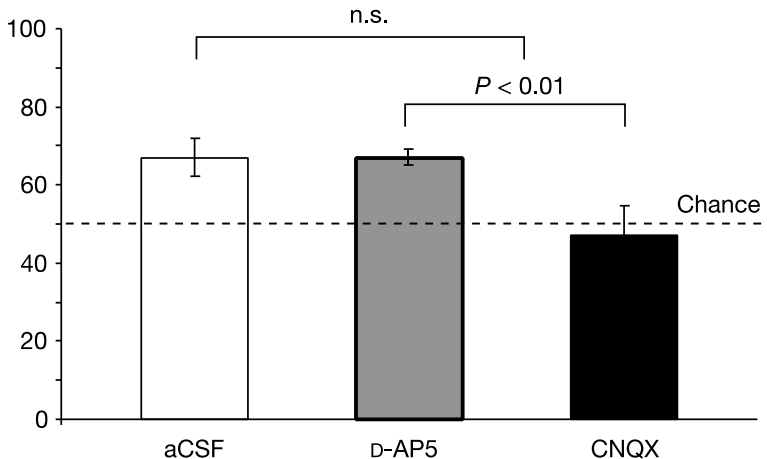

d, Drug-infusions before sample trials revealed an overall effect of drug ( $F=4.61$, d.f. $2 / 20, P<0.025)$. There was better-than-chance and above-chance performance on aCSF days than on drug days $(F=5.30$, d.f. $1 / 20, P<0.01 ; t=3.86$, d.f. 10 ,

$P<0.003)$, and no difference between D-AP5 and CNQX treatment days $(F<1)$, which were both at chance. e, Drug infusions before choice trials also revealed an overall drug effect $(F=4.38$, d.f. $2 / 20, P<0.03)$, no difference between days with aCSF and D-AP5 treatment $(F<1)$, but better-than-chance and above-chance performance on these days compared with those with CNQX infusions $(F=10.76$, d.f. 1/20, $P<0.01$; aCSF $t=3.87$, d.f. $10, P<0.003$; D-AP5 $t=4.7$, d.f. $10, P<0.001$ ) 
locations with performance significantly above chance $(t=3.73$, d.f. $1 / 11, P<0.003)$. We also routinely conducted the 'positive control' of establishing electrophysiological inactivation of the hippocampus in other rats by using the same vial of CNQX as used behaviourally. It follows that the animals are not 'lost in space' when the hippocampus is inactivated and can retrieve the spatial locations of repeat-trial cued flavours.

Our experiments establish that rats can, in one trial, encode a novel association between a food flavour ('what') and a spatial location ('where') and can preferentially recall the correct location when cued with the flavour item of the paired associate. Previous studies of paired-associate learning in animals have used multi-trial training procedures and recognition tests ${ }^{13,16,17}$. Here, cued recall after only one encoding trial is not an artefact of cryptic odour guidance, because performance was above chance during nonrewarded probe tests. Choice cannot be guided by recognition memory or relative recency as in the widely used task involving delayed non-matching to sample ${ }^{18,19}$, because the two flavours and locations used as samples on a given day were arranged to be equally familiar and counterbalanced with respect to order. Any differential preference for one flavour over the other was fully controlled by counterbalancing, with the miscue test establishing that the rats would choose equally between the two samples when cued with a different flavour. Thus, the event arena offers a valid test of

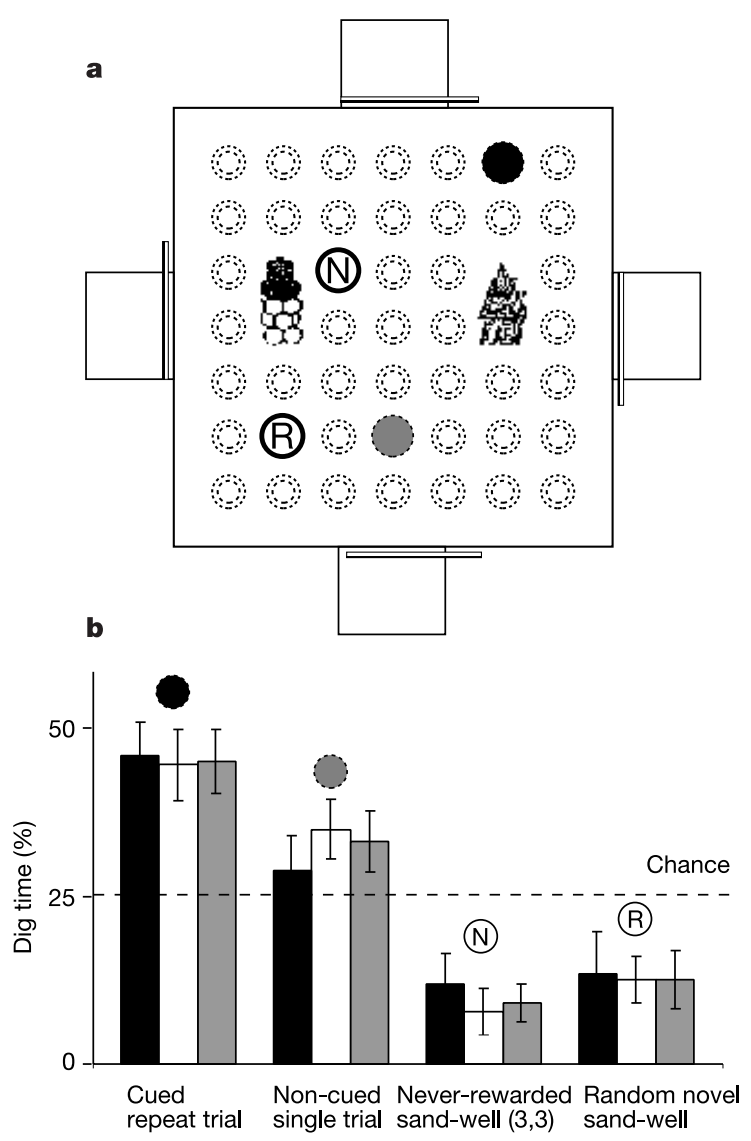

Figure 4 Insensitivity of repeat-trial paired associates to hippocampal inactivation. a, Event arena with representative layout of four sand-wells used during non-rewarded probe tests: a cued repeat-trial location (for example (1,5); black circle), a non-cued single-trial location (grey circle), the never-rewarded location, $(3,3)(\mathrm{N})$, and a random novel location (R). $\mathbf{b}$, Different proportions of digging were observed at the four sand-wells ( $F=26.1$, d.f. $2 / 24, P<0.0001$ ), but intrahippocampal CNQX (black bars) did not disrupt performance $(F<1)$. The cued repeat-trial location was above chance $(P<0.005)$; the never-rewarded location was below chance $(P<0.005)$. White bars, aCSF; grey bars, no infusion. associative cued recall in animals ('what-where' in the terminology of ref. 2).

Our neurobiological findings indicate that blocking hippocampal NMDA receptors during sample trials impairs the memory encoding of paired associates but has no effect on retrieval. We suspect that the effect on encoding is primary, with information about the 'what' and 'where' of single events associated in hippocampal circuitry, although a cascade of NMDA-receptor-dependent components of event memory might be involved. Earlier studies with a strictly spatial paradigm emphasized the event-like nature of onetrial spatial memory ${ }^{14}$. Pairing of events with familiar places could work if recall of the spatial information, from a neocortical site of storage, was by means of the direct input to CA1 from the entorhinal cortex ${ }^{20}$, with event processing upstream in hippocampal circuity. Blocking hippocampal fast synaptic transmission impairs both encoding and retrieval, a finding compatible with activations seen during human functional imaging ${ }^{21}$ and experience-induced strengthening of transmission by means of AMPA-receptor insertion $^{22}$. However, the interval between sample and recall trials was too short to realize a firm dissociation. Finally, if a flavour-place pair is trained repeatedly, accurate retrieval is still possible when the hippocampus is inactivated. The rats will most probably do this by accessing a consolidated neocortical memory trace of the paired associate rather than by recalling the events of the earlier sample trials by means of the now-inaccessible hippocampal trace. Speculatively, this is akin to the distinction in human studies between 'knowing' and 'remembering'23, but a literal application of this distinction to animals is premature. The protocol's flexibility could make it valuable as an animal analogue of a diagnostic test for mild Alzheimer's disease ${ }^{24}$ and to analyse the neural mechanisms of episodic-like memory including, in due course, the incorporation of temporal and sequential factors ${ }^{3,4,25}$.

\section{Methods}

Subjects

Male Lister hooded rats (initially 3 months old) were maintained on water ad libitum but a restricted diet to maintain a body weight at $85 \%$ of the free-feeding weight. Procedures complied with the UK Animals (Scientific Procedures) Act, PPL 60/2484.

\section{Apparatus}

The arena was made of clear Perspex ( $1.6 \mathrm{~m} \times 1.6 \mathrm{~m}, 0.3 \mathrm{~m}$ side walls). The four start boxes $(0.3 \mathrm{~m} \times 0.3 \mathrm{~m})$ were placed centrally in each wall, with sliding doors for arena access. The 49 food wells ( $7 \mathrm{~cm}$ diameter, $4 \mathrm{~cm}$ deep) were normally covered by an insert made of white Perspex, the same colour as the floor, to ensure an unbroken surface. Two unused locations (row 4, column 2-(4,2) - and (4,6)) were covered by distinctive landmarks $20 \mathrm{~cm}$ high (a pagoda and a glued stack of golf balls). The sand inside each sand-well was slightly adulterated by ground-up rat chow of all flavours, thoroughly stirred, and renewed regularly. One or more sand-wells could be exposed on sample or choice trials and, if rewarded, contained a single 1-g pellet of flavoured rat chow. The 28 flavours available included: a base flavour; single flavours such as almond, anise and apple; and combination flavours (manufactured as a single pellet) such as banana plus cherry (full list in Supplementary Information).

\section{Training procedure}

The animals were observed by means of two video cameras connected to video recorders and computer software for tracking (videos of the animals performing the task are available at http://neuroweb-2.dns.ed.ac.uk/video/)

In habituation experiments, on each of five days the rats explored the arena, initially found food on top of open sand-wells and then learned to dig for food pellets that were gradually hidden under the sand.

Training was as described in the text for 5 days per week; two flavours were used on sample trials and, typically, one only of these two flavours was available at the choice trial.

In experiment 1 , different flavours were used on successive days and, when reused after 10-15 days, were always in different flavour-location combinations. Sample trials were rewarded with 1-g food pellets. The reminder cue in the start box on choice trials was $500 \mathrm{~g}$ and choice trials were rewarded with 1-g food pellets (allowing correction). Training continued over 8 months. The first phase of training monitored first choices on rewarded cued-recall choice trials $(n=8)$ preceded by single-presentation or double-presentation sample trials (see Supplementary Information). We then switched all rats ( $n=16$, using only the 12 rats that had learned the task) to single-presentation sample trials for 15 days before interspersing non-rewarded probe tests with rewarded choice trials. These probe tests permitted measures of digging times on both cued-recall and miscued-recall choice trials with five dig locations. Digging times were averaged over two non-rewarded days, enabling counterbalancing with respect to the sample flavour that was cued at recall. In 
examining blocking of hippocampal AMPA and NMDA receptors with CNQX, D-AP5 and aCSF, drugs were applied $15 \mathrm{~min}$ before sample trials or $15 \mathrm{~min}$ before choice trials (one day for each of six conditions), with a 20-min interval between sample and choice trials. In-house software (LabView) was used to monitor digging.

Experiment $2(n=12)$ was conducted with new animals, with two flavour-place pairings repeated amid other novel pairs. After habituation, the rats were given extended training ( 5 days per week for 8 weeks): In any week, one day involved sample presentations and then a choice trial of the repeated flavour-location pairs (apple in location $(1,5)$; brandy in location $(7,5)$ ); one day only novel pairs; and three days with mixed pairs (for example a novel pair for sample 1 and apple in $(1,5)$ for sample 2 , or brandy, or vice versa). Over 8 weeks, the animals received 20 sample presentations of the repeat-trial pairs $(8 \times 2.5)$. The choice trials always had four sand-wells open: the cued location, the noncued but previously sampled location, a never-rewarded location, $(3,3)$, and a novel location. Probe tests followed during weeks 9-12 of testing, including CNQX, aCSF or no-drug conditions, these being interspersed with further training days. Four sand-wells were also used in these probe tests (see Fig. 4)

\section{Surgery, electrophysiology and drug infusions}

Stainless-steel guide cannulae (26-gauge) were implanted into the dorsal hippocampus bilaterally using standard stereotaxic techniques under tribromoethanol anaesthesia. Electrophysiological studies to calculate the CNQX and D-AP5 doses used separate animals under urethane anaesthesia $\left(1.5 \mathrm{~g} \mathrm{~kg}^{-1}\right)$. Dentate field potentials were monitored unilaterally by means of a recording electrode $(75 \mu \mathrm{M})$ lowered into the hilus, with a bipolar stimulating electrode placed into the angular bundle of the perforant path. The concentric bipolar recording electrode (Rhodes) was $1,400 \mu \mathrm{m}$ from the infusion cannulae. The slope, amplitude and population spike of the field potentials was monitored with in-house software (LabView). Drug infusions in these and the behavioural experiments were $1 \mu \mathrm{l} \mathrm{CNQX} \mathrm{(3} \mathrm{mM;} \mathrm{Tocris)} \mathrm{and} 1 \mu \mathrm{l}$ D-AP5 (30 mM; Tocris) per cannula. The infusion cannula was fixed for the electrophysiological experiments, but was left in place for $1 \mathrm{~min}$ after infusion for the behavioural experiments, with the animal lightly restrained in the hand of one of the experimenters (M.D. or R.L.)

Received 17 December 2002; accepted 29 April 2003; doi:10.1038/nature01769.

1. Tulving, E. Elements of Episodic Memory (Oxford Univ. Press, New York, 1983)

2. Clayton, N. S. \& Dickinson, A. Episodic-like memory during cache recovery by scrub jays. Nature 395 272-274 (1998).

3. Fortin, N. J., Agster, K. L. \& Eichenbaum, H. B. Critical role of the hippocampus in memory for sequences of events. Nature Neurosci. 5, 458-462 (2002).

4. Kesner, R. P., Gilbert, P. E. \& Barua, L. A. The role of the hippocampus in memory for the tempora order of a sequence of odors. Behav. Neurosci. 116, 286-290 (2002).

5. Bliss, T. V. P. \& Lømo, T. Long-lasting potentiation of synaptic transmission in the dentate area of the anaesthetized rabbit following stimulation of the perforant path. J. Physiol. (Lond.) 232, 331-356 (1973).

6. Martin, S. J., Grimwood, P. D. \& Morris, R. G. M. Synaptic plasticity and memory: An evaluation of the hypothesis. Аnпu. Rev. Neurosci. 23, 649-711 (2000).

. Morris, R. G. M. \& Frey, U. Hippocampal synaptic plasticity: Role in spatial learning or the automatic recording of attended experience? Phil. Trans. R. Soc. Lond. B 352, 1489-1503 (1997).

8. Eichenbaum, H., Dudchenko, P. A., Wood, E. R., Shapiro, M. \& Tanila, H. The hippocampus, memory and place cells: Is it a spatial memory or a memory space? Neuron 23, 209-226 (1999).

9. Bleakman, D. \& Lodge, D. Neuropharmacology of AMPA and kainate receptors. Neuropharmacology 37, 1187-1204 (1998)

10. Bliss, T. V. \& Collingridge, G. L. A synaptic model of memory: Long-term potentiation in the hippocampus. Nature 361, 31-39 (1993).

11. Cowan, W. M., Sudhof, T. C. \& Stevens, C. F. Synapses (Johns Hopkins Univ. Press, 2001).

12. O'Keefe, J. \& Nadel, L. The Hippocampus as a Cognitive Map (Clarendon Press, Oxford, 1978).

13. Gilbert, P. E. \& Kesner, R. P. Role of the rodent hippocampus in paired-associate learning involving associations between a stimulus and a spatial location. Behav. Neurosci. 116, 63-71 (2002).

14. Steele, R. J. \& Morris, R. G. M. Delay-dependent impairment of a matching-to-place task with chronic and intrahippocampal infusion of the NMDA-antagonist D-AP5. Hippocampus 9, 118-136 (1999).

15. Kim, J. J. \& Fanselow, M. S. Modality-specific retrograde amnesia of fear. Science 256, 675-677 (1992)

16. Sakai, K. \& Miyashita, Y. Neural organization for the long-term memory of paired associates. Nature 354, 152-155 (1991)

17. Gaffan, D. Scene-specific memory for objects: A model of episodic memory impairment in monkeys with fornix transection. J. Cogn. Neurosci. 6, 305-320 (1994).

18. Mishkin, M. \& Delacour, J. An analysis of short-term visual memory in the monkey. J. Exp. Psychol.: Anim. Behav. Processes 1, 326-334 (1975).

19. Zola-Morgan, S. \& Squire, L. R. Medial temporal lesions in monkeys impair memory on a variety of tasks sensitive to human amnesia. Behav. Neurosci. 99, 22-34 (1985).

20. Brun, V. H. et al. Place cells and place recognition maintained by direct entorhinal-hippocampal circuitry. Science 296, 2243-2246 (2002)

21. Zeineh, M. M., Engel, S. A., Thompson, P. M. \& Bookheimer, S. Y. Dynamics of the Hippocampus during encoding and retrieval of face-name pairs. Science 299, 577-580 (2003).

22. Takahashi, T., Svoboda, K. \& Malinow, R. Experience strengthening transmission by driving AMPA receptors into synapses. Science 299, 1585-1588 (2003).

23. Gardiner, J. M. in Episodic Memory: New Directions in Research (eds Baddeley, A., Conway, M. \& Aggleton, J.) 11-30 (Oxford Univ. Press, 2001).

24. Swainson, R. et al. Early detection and differential diagnosis of Alzheimer's disease and depression with neuropsychological tasks. Dement. Geriatr. Cogn. Disord. 12, 265-280 (2001).

25. Lisman, J. E. Relating hippocampal circuitry to function: Recall of memory sequences by reciprocal dentate-CA3 interactions. Neuron 22, 233-242 (1999).

Supplementary Information accompanies the paper on www.nature.com/nature.
Acknowledgements We thank N. Clayton, D. Donaldson, P. Dudchenko, L. Jacobs, E. Moser and E. Wood for discussion. Funded by MRC Innovation and Programme Grants and an E.U. Framework V Grant.

Competing interests statement The authors declare that they have no competing financial interests.

Correspondence and requests for materials should be addressed to R.G.M.M

(r.g.m.morris@ed.ac.uk).

\section{T-type calcium channel regulation by specific G-protein $\beta \gamma$ subunits}

\author{
Joshua T. Wolfe, Hongge Wang, Jason Howard, James C. Garrison \\ \& Paula Q. Barrett
}

Department of Pharmacology, University of Virginia, Charlottesville, Virginia 22908, USA

Low-voltage-activated (LVA) T-type calcium channels have a wide tissue distribution and have well-documented roles in the control of action potential burst generation and hormone secretion ${ }^{1}$. In neurons of the central nervous system and secretory cells of the adrenal and pituitary, LVA channels are inhibited by activation of G-protein-coupled receptors that generate membrane-delimited signals $^{2-5}$, yet these signals have not been identified. Here we show that the inhibition of $\alpha_{1 H}\left(\mathrm{Ca}_{\mathrm{v}} 3.2\right)$, but not $\alpha_{1 \mathrm{G}}\left(\mathrm{Ca}_{\mathrm{v}} 3.1\right) \mathrm{LVA}$ $\mathrm{Ca}^{2+}$ channels is mediated selectively by $\beta_{2} \gamma_{2}$ subunits that bind to the intracellular loop connecting channel transmembrane domains II and III. This region of the $\alpha_{1 \mathrm{H}}$ channel is crucial for inhibition, because its replacement abrogates inhibition and its transfer to non-modulated $\alpha_{1 \mathrm{G}}$ channels confers $\beta_{2} \gamma_{2}$-dependent inhibition. $\beta \gamma$ reduces channel activity independent of voltage, a mechanism distinct from the established $\beta \gamma$-dependent inhibition of non-L-type high-voltage-activated channels of the $\mathrm{Ca}_{\mathrm{v}} 2$ family $^{6,7}$. These studies identify the $\alpha_{1 \mathrm{H}}$ channel as a new effector for G-protein $\beta \gamma$ subunits, and highlight the selective signalling roles available for particular $\beta \gamma$ combinations.

G-protein $\beta \gamma$ subunits released by receptor activation have important physiological functions in the regulation of G-proteingated, inward-rectifier potassium $(\mathrm{GIRK})^{8}$; and $\mathrm{Ca}_{\mathrm{v}} 2 \mathrm{Ca}^{2+}$ (refs 8, 9) channels (N-type, P/Q-type and R-type), by interacting with intracellular domains of the channel molecules. In the $\mathrm{Ca}_{\mathrm{v}} 2$ family, these domains include the I-II cytoplasmic loop and the amino and carboxy termini ${ }^{10-12}$. The effects of $\beta \gamma$ inhibition on the $\mathrm{Ca}_{\mathrm{v}} 2$ family members are also modulated by protein kinase $C$ (ref. 13) and the synaptic proteins syntaxin 1A, SNAP-25 and cysteine-string protein $^{14}$. By contrast, neither the identity of the G-protein subunit mediating the inhibition of LVA channels nor the domain of the LVA channel supporting inhibition is known.

We tested the possibility that G-protein $\beta \gamma$ subunits could regulate whole cell LVA currents, comparing the effects of $\beta_{2} \gamma_{2}$ and $\beta_{1} \gamma_{2}$ overexpression in HEK-293 cells stably expressing $\alpha_{1 \mathrm{H}}$ $\mathrm{Ca}^{2+}$ channels (Fig. 1a, upper panels). At all potentials tested, $\alpha_{1 \mathrm{H}}$ channels are specifically inhibited by $\beta_{2} \gamma_{2}$ dimers, decreasing current density by $50 \%$ (Fig. 1 b, left panel). Surprisingly, the $\beta_{1} \gamma_{2}$ dimer did not inhibit the channel. This difference in inhibitory selectivity between the two $\beta$ subunits could not be attributed to inadequate levels of $\beta_{1} \gamma_{2}$ protein expression. Immunoblots of the transfected cell lysates (Fig. 1b, inset) show that the expression of green fluorescent protein (GFP), a marker for transfection efficiency, is similar in both cases, and that each Flag-tagged $\beta$ subunit 